Adıyaman Üni. Sağlık Bilimleri Derg, 2015; 1(3):162-168

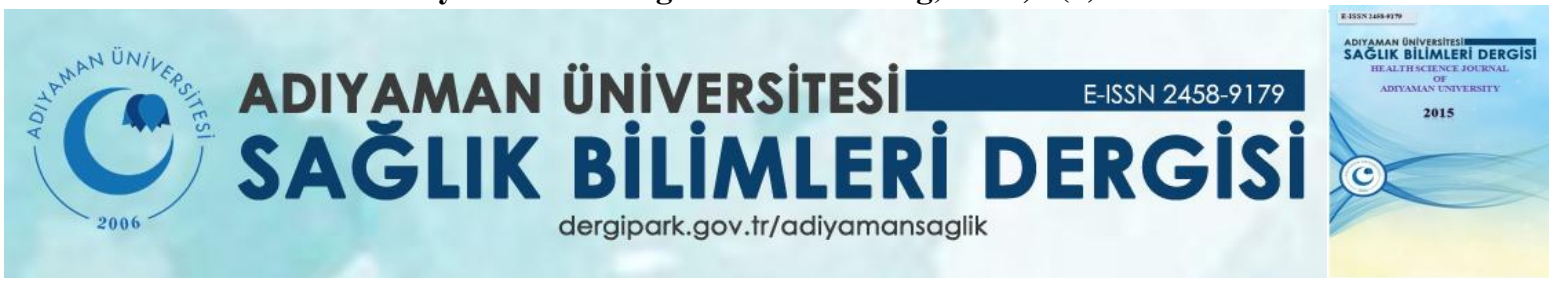

Olgu sunumu/ Case report

\title{
Acil Serviste Pantopaque Kullanımına Bağlı Farklı Bir İntraserebral Görüntü; Pantopaque Droplets. \\ İrfan AYDIN ${ }^{1}$, M.Kaan POYRAZ ${ }^{1}$, Melahat POYRAZ², Abdullah ALGIN ${ }^{1}$ \\ ${ }^{1}$ Adıyaman Üniversitesi Tıp Fakültesi Acil Tıp Anabilim Dalı \\ ${ }^{2}$ Adıyaman Üniversitesi Tıp Fakültesi Radyoloji Anabilim Dalı.
}

ÖZ

Bel ağrıların tanısında manyetik resonans görüntüleme kullanılmadan önce radyografi ve miyelografi kullanılıyordu.Myelografi, Omuriliğe kontrast madde verilerek çekilen omurilik röntgenidir. Pantopaque geçmişte myelografi amacıyla kullanılmış olan yağlı bir kontrast maddedir. Etken maddesi iodophenylundecylic asittir. En önemli yan etkisi subaraknoid mesafelerde damlacık şeklinde kalıntılar bırakmasıdır. Günümüzde yerini tamamen suda eriyen kontrast maddelere bırakmıştır. Bu yazıda acil servisimize baş ağrısı nedeniyle başvuran ve subaraknoid bölgede pantopaque'ye bağlı yağ damlacıkları olan bir vaka sunumu yaptık.

Anahtar Kelimeler: Acil servis, pantopaque, beyin tomografisi.

\begin{tabular}{l}
\hline Yazışmadan Sorumlu Yazar \\
\hline İfan AYDIN \\
Adıyaman Üniversi Tıp Fakültesi \\
Acil Tıp Anabilim Dalı. \\
Tel : +90 05062244804 \\
Email: irfanaydinyyu@yahoo.com.tr \\
\hline
\end{tabular}

\begin{tabular}{ll}
\hline Geliş Tarihi: & 12.09 .2017 \\
\hline Kabul Tarihi: & 30.11 .2017
\end{tabular}




\title{
Unusual Cause of Hyperdensity on CT images of The Brain in the Emergency \\ Department : Pantopaque Droplets
}

\begin{abstract}
Radiography and myelography were used in the diagnosis of lumbar pain before magnetic resonance imaging. Myelography is a type of radiographic examination that uses a contrast medium to detect pathology of the spinal cord which causes back pain. Pantopaque which contain iodophenylundecylic acid is a contrast matter that has been used for myelography in the past. The most important side effect is to leave droplet-like residues in the subarachnoid space. Nowadays water-soluble contrasting materials are used for myelography instead of oil-based pantopaque. We present a case report which has dropletlike residues in the subarachnoid space after myelography.
\end{abstract}

Keywords: Emergency, pantopaque, brain tomography. 


\section{GİRIŞ}

1960’ların ortasında spinal kanalın içeriğini analiz etmek için myelografi kullanılıyordu. Myelografi çekiminde de kontrast madde olarak omiriliğe pantopaque (iophendylate, a fatty acid) enjekte ediliyordu. Pantopaque'nin nonabsorbabl ve yağlı bir çözelti olmasından faydalanılıyordu (1,4). 1980'lere kadar Avrupa'da ve Kuzey Amerika'da yaygın olarak kullanıldı (2). Fakat 1980'den sonra pantopaque kullanımını takiben, çok sayıda komplikasyon bildirildi. Bu nedenle yerini suda çözünen kontrast maddelere bıraktı $(3,5)$. Bizde Acil servise başvuran ve hikayesinde 30 yıl önce belinden renkli film çekildiğini söyleyen böyle bir hastanın beyin bilgisayarlı tomografisi ve magnetik resonans görüntülerini litaratür eşliğinde sunmayı amaçladık.

\section{OLGU}

67 yaşında bayan hasta sol dizden aşağısını hissetmeme ve üzerine basamama şikayeti ile acil servisimize başvurdu.Vital bulguları stabil olan hastanın yapılan fizik muayenesinde her iki bacak duyu muayenesi doğaldı. Motor kuvvet muayenesi 4/5 olarak değerlendirildi. Babinski refleksi bilateral negatifti. Hastanın özgeçmişinde 30 yıl önce bel ağrısı nedeniyle belinden ilaç verilip (Pantopaque) film çekildiği öğrenildi. Bunun dışında özgeçmiş ve soy geçmişinde bir özellik olmayan hastaya serebrovasküler hastalık ön tanısı ile Beyin Tomografisi çekildi. Beyin Tomografisinde özellikle serebellum düzeyinde beyin sap1 çevresinde ve temporal frontal loblar düzeyinde multiple milimetrik boyutlu hiperdens görünümler izlendi. Bunların uygulanmış kontrast madde enjeksiyonuna bağlı olduğu tespit edildi (Resim 1). Hastanın Beyin Magnetik Resonans (MRG) görüntülerinde de; bilateral serebellar hemisferlerde, 4. Ventrikül sağ yan komşuluğunda, pons etrafında, sağ temporalde, interhemisferik fissür anteriorunda T1A sekansta hiperintens, diğer sekanslarda sinyalsiz milimetrik lezyonlar tespit edildi (Resim 2). Hastanın lomber MRG, beyin tomografisi ve 
kliniği göz önüne alındığında miyelografi sonrası araknoidit ile uyumlu olduğu görüldü

(Resim 3). Bunun üzerine hastaya pantopaque droplets tanısı konuldu. Bunun dışında herhangi bir acil patoloji saptanmayan hasta nöroloji poliklinik kontrolü önerilerek taburcu edildi.

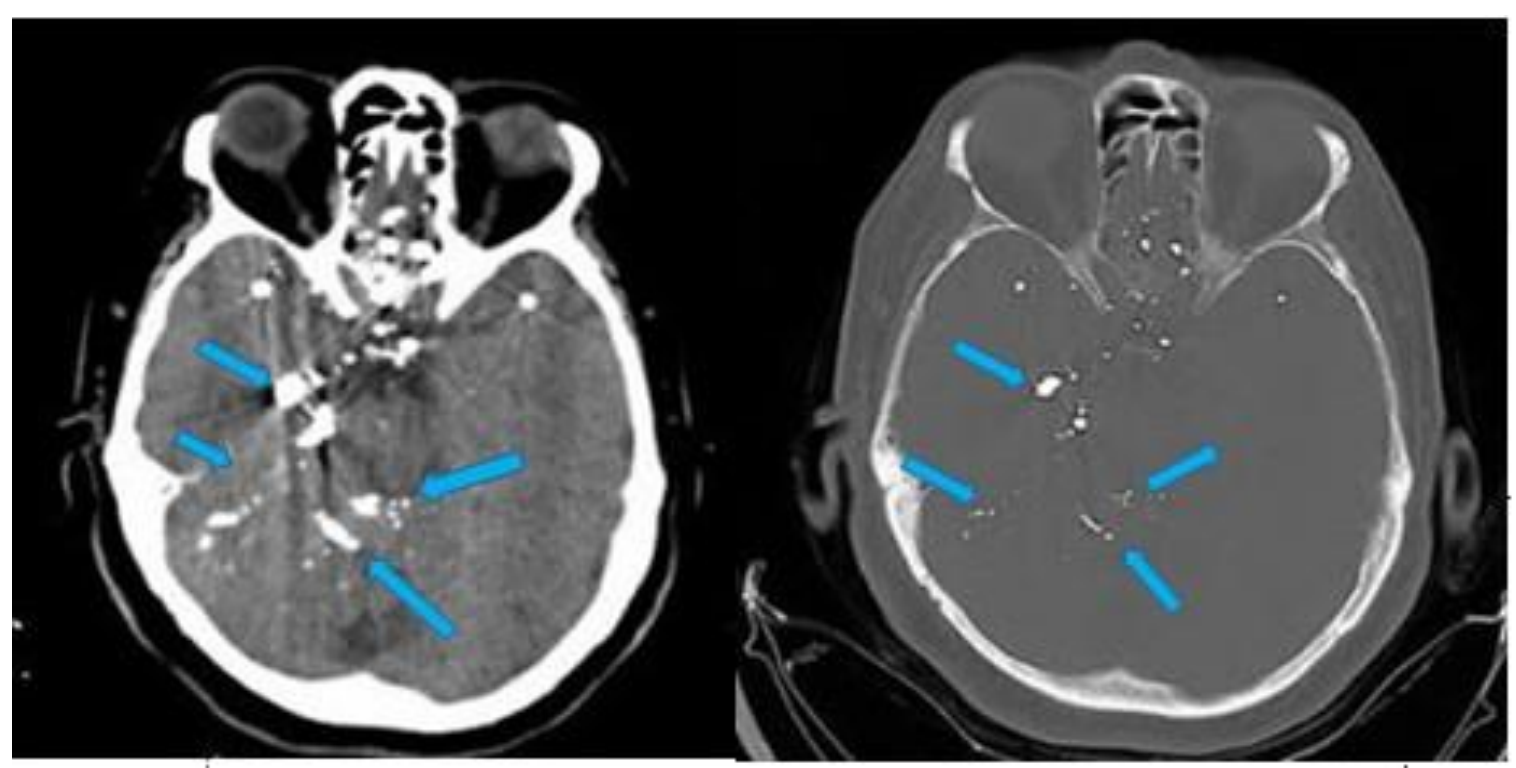

Resim 1. Multiple milimetrik boyutlu hiperdens görünümler.

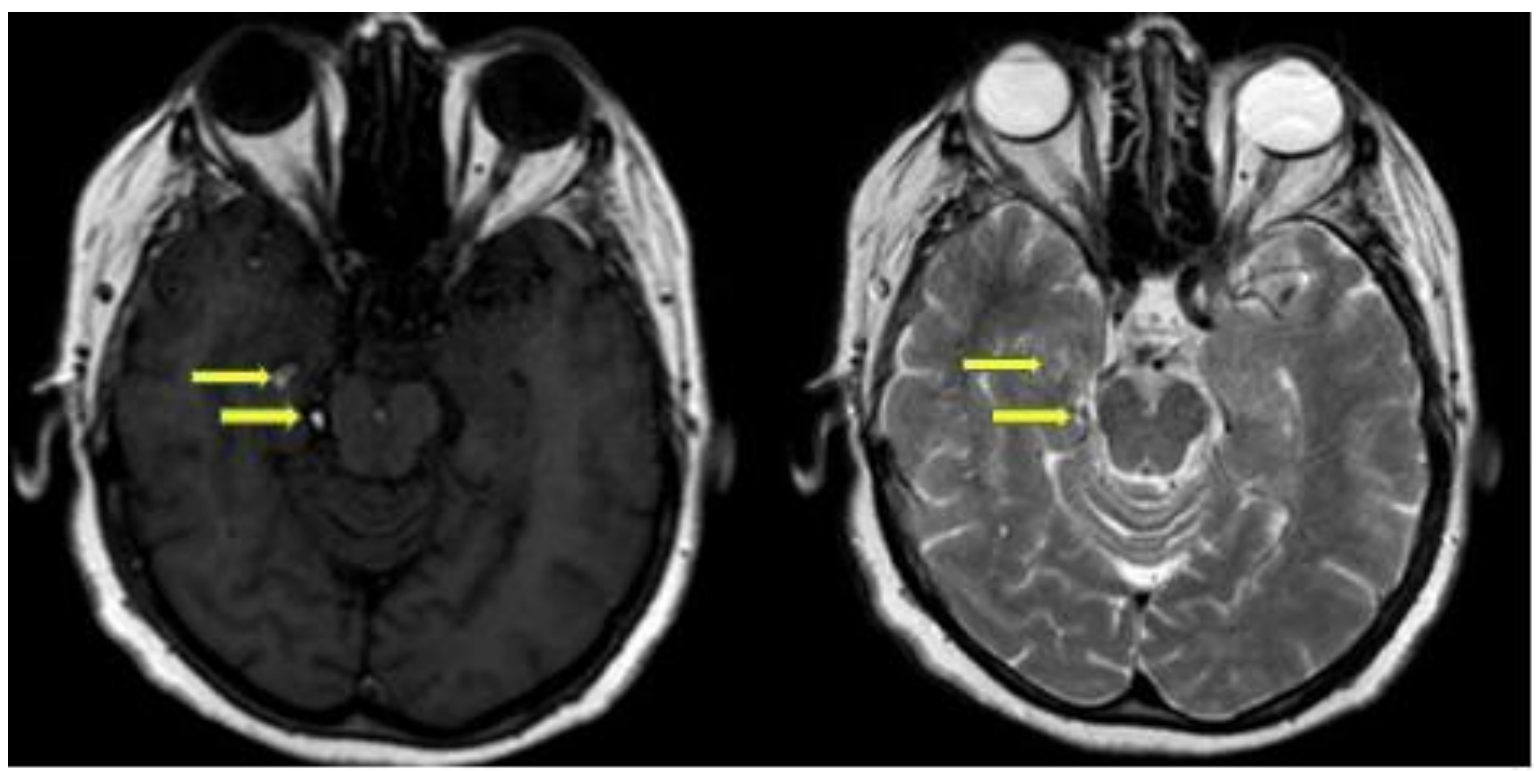

Resim 2. Magnetik Resonans (MR) görüntülerinde T1A sekansta hiperintens ve T2A da hipointens lezyonlar. 


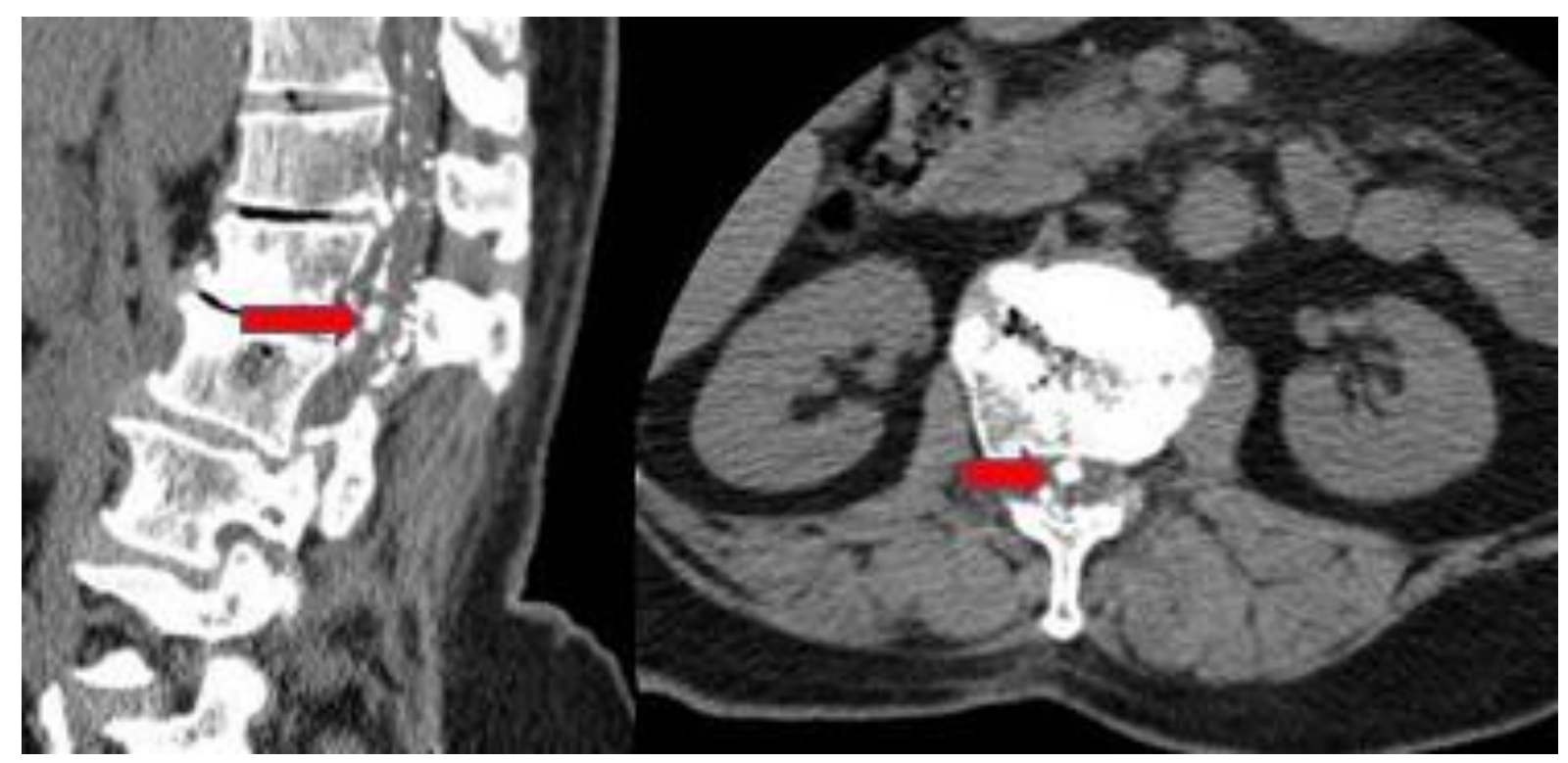

Resim 3. Lomber Tomografi görüntülerinde hiperdens görünümler.

\section{TARTIŞMA}

Myelografi 1919 y1lından sonra 1980’lere kadar tüm dünyada vertebral kanal patolojilerinde tanısal amaçlı kullanılan bir tekniktir (4).EthyIiodophenyIundecyIate (pantopaque,myodil) 1941'den sonra myelografi çekimi için kontrast madde olarak kullanılmıştır (5). Myodil'de pantopaque gibi 1980'lere kadar yaygın olarak kullanılmıştır. Fakat bu yağlı kontrast madde beyin omurilik sıvısından çok yavaş bir şekilde elimine olduğu, 6 gün içinde kistleştiği ve yılda $0,5-3$ cc kadar beyin omurilik sıvısından temizlendiği bilinmektedir. $\mathrm{Bu}$ süreç de araknoid zarda irritasyona ve sonrasında kronik araknoidit gelişmesine zemin hazırlar (6).

Myelografi'de kullanılan pantopaque/Myodil'e bağlı olarak literatürde, araknoid adhezyonlar, kistler, spinal kordta fistül oluşumu, kord basısı ve sinir kökü irritasyonu 
bildirilmiştir (8). Residuel miyodil ve pantopaque'nin MRG'ye dayalı ayırıcı tanısında lipomlar, hemanjiom, hemoraji ve melanomlar yer alır (9). Ülkemizde Paşaoğlu ve ark. tarafindan köpekler üzerinde pantopaque,iopamidol ve iohexol ile myelografi çalışması yapılmıştır. Bu çalışma sonucu sadece pantopaque'ye bağlı araknoidal reaksiyonun oluştuğu tespit edilmiştir (10). Myint ve ark.yaptığı bir çalışmada da vertebral fraktür nedeniyle takip edilen bir hastanın MRG görüntülerinde de tümör zannedilen pantopaque kalıntısı bulmuşlardır (11). Bu yağlı kontrast maddeler uzun yıllar kullanılmış olmakla birlikte, 1980’lerden sonrada yerini suda çözünen kontrast maddelere bırakmıştır (7).

Benzer şekilde sunmuş olduğumuz bu çalışmadaki olguda da myelografi öyküsü ve çekilen BT ve MRG de pantopaque kalıntısı ile uyumlu görüntüler tespit edildi.

Sonuç olarak; acil servislerde herhangi bir nedenle çekilen Tomografi veya MR görüntülemede bu tür vakaların görülmesi doktorun kafasını karıştırmakta ve tanıyı zorlaştırmaktadır. Bu nedenle özellikle özgeçmiş hikayesinin derinleştirip 20-40 yıl öncesine gidilmesi ve sırtına enjeksiyon veya ilaçlı film çekilip çekilmediğinin sorgulanmasının doktora daha çok yardımcı olacağı kanısındayız. 


\section{KAYNAKLAR}

1. Pawl R. History of myelography with pantopaque contributing to arachnoiditis. Surgical neurology international $2014: 5 ; 315$.

2. Gnanalingham K.K, Joshi SM, and Sabin L. Thoracic arachnoiditis, arachnoid cyst and syrinx formation secondary to myelography with Myodil, 30 years previously. European Spine Journal 2006: 15(5);661-663.

3. Lee SK,Kim DH,Lim DJ. Asymptomatic thoracic Pantopaque cyst mimicking an intradural extramedullary lipoma on MR images. European Spine Journal 2013: 22 (3); 321-328.

4. Michael SM, Raaf J. Complications of pantopaque myelography. Case report and review. Journal of neurosurgery 1962: (19);302-311.

5. Ramsey GH, French JD, Strain WH. Iodinated Organic Compounds as Contrast Media for Radiographic Diagnoses: IV. Pantopaque Myelography 1.Radiology 1944: 43(3 );236-240.

6. Wang SC, Lu PS, Wu PW, Yeh CH, Wang CJ, Chang CC. Intracranial migration of iophendylate four decades after conventional myelography.British Journal of Neurosurgery 2016 Dec:1:1-2.

7. Hugh AE. The subdural space of spine: a lymphatic sink? Myodil's last message. Clin Anat. 2010;23(7):829-39.

8. Barsoum AH, Cannillo KL. Thoracic constrictive arachnoiditis after Pantopaque myelography: report of two cases. Neurosurgery 1980;6(3):314-6

9. Tabor EN, Batzdorf U. Thoracic spinal pantopaque cyst and associated syrinx resulting in progressive spastic paraparesis: case report. Neurosurgery 1996;39(5):1040-2.

10. Pasaoglu A, Gök A, Patıroğlu TE. An experimental evaluation of response to contrast media: Pantopaque, iopamidol, and iohexol in the subarachnoid space. Investigative radiology 1988: 23(10);762-766.

11. Myint OO,Wang Z,Sakakibara T,Kasai Y. Magnetic resonance imaging findings of remnants of an intradural oil-based contrast agent: report of a case.The journal of spinal cord medicine 2012: 35(3);187-190. 